\title{
Research on treating processes of Nickel Laterite Ores in the world and Vietnam
}

\author{
Khanh Tuan Nguyen ${ }^{1}$, Toi Trung Tran ${ }^{2,}$, , Thuat Tien Phung ${ }^{2}$ \\ ${ }^{1}$ Southern Branch of the Vietnam Institute ofGeosciences and Minerals, Ho Chi Minh City, Vietnam \\ ${ }^{2}$ Hanoi University of Mining and Geology, Hanoi, Vietnam
}

\begin{abstract}
ARTICLE INFO
ABSTRACT

Article history:

Received 18 th Jan. 2021

Revised 14 $4^{\text {th }}$ May 2021

Accepted $15^{\text {th }}$ June 2021

Keywords:

Magnetic separation,

Nickel laterite ores,

Reduction roasting,

Thanh Hoa.

Laterite nickel ores, accounting for about 70\% of total world nickel reserves, are very abundant and considered as an important resource of nickel. However, nickel content of laterite ores are generally low of about $0.5 \div 2.5 \% \mathrm{Ni}$. In addition, nickel minerals are very finely disseminated in the ores, so that traditional separation methods such as froth flotation, gravity method, magnetic method, and electrical separation produce very low recovery efficiency. Currently, the treatment of this type of ores is being intensively studied and directed to use common available processes including: Hydrometallurgical, pyrometallurgical, and reduction roasting - magnetic separation processes. This article aims to summarize typical studies on the characteristics of current laterite nickel ore processing technologies commonly used in the world and in Vietnam. From the review, appropriate direction for treatment of Thanh Hoa - Vietnam laterite nickel ores can be proposed.
\end{abstract}

Copyright (C) 2021 Hanoi University of Mining and Geology. All rights reserved.

${ }^{*}$ Corresponding author

E - mail: trantrungtoi@humg.edu.vn

DOI: 10.46326/JMES.2021.62(3b).05 


\title{
Tạp chí Khoa học Kỹ thuật Mỏ - Địa chất
}

Trang điện tử: http://tapchi.humg.edu.vn

\section{Các nghiên cứu về quy trình xử lý quặng niken laterit trên thế giới và ở Việt Nam}

\author{
Nguyễn Tuấn Khanh ${ }^{1}$, Trần Trung Tới ${ }^{2,}{ }^{*}$, Phung Tiến Thuật ${ }^{2}$ \\ ${ }^{1}$ Phân viện Khoa học Địa chất và Khoáng sản phía Nam, TP. Hồ Chí Minh, Việt Nam \\ 2 Trường Đại học Mỏ - Địa chất, Hà Nội, Việt Nam
}

\begin{tabular}{|c|c|}
\hline THÔNG TIN BÀI BÁO & TÓM TẮT \\
\hline $\begin{array}{l}\text { Quá trình: } \\
\text { Nhận bài 18/01/2021 } \\
\text { Sứa xong 14/5/2021 } \\
\text { Chấp nhận đăng 15/6/2021 } \\
\text { Tù̀ khóa: } \\
\text { Quặng niken laterit, } \\
\text { Tuyển từ, } \\
\text { Thanh Hóa. } \\
\text { Thiêu hoàn nguyên, }\end{array}$ & 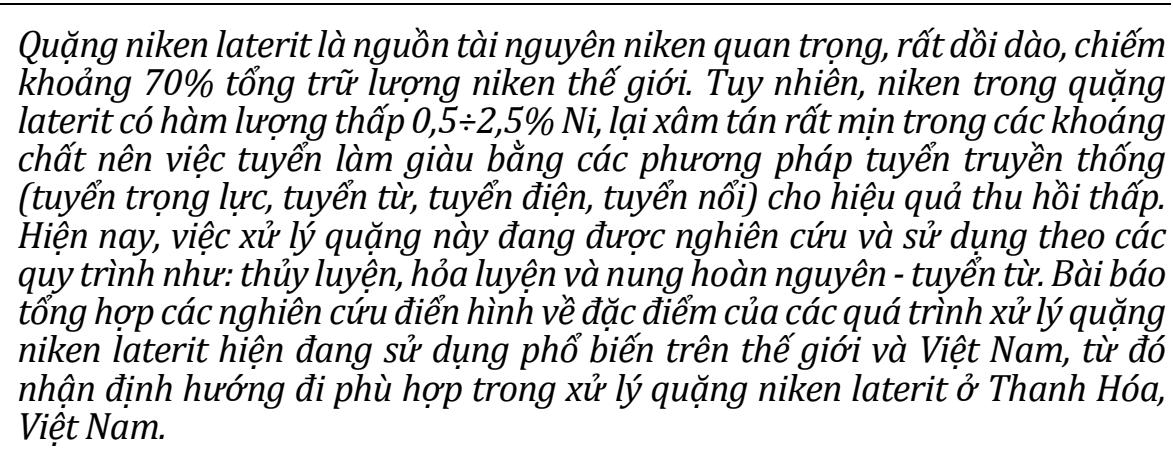 \\
\hline
\end{tabular}

C 2021 Trường Đại học Mỏ - Địa chất. Tất cả các quyền được bảo đảm.

\section{Mở đầu}

Niken là một kim loại màu thiết yếu và được sử dụng rộng rãi cho thép không gỉ và thép hợp kim, mạ điện hoặc làm chất xúc tác trong quá trình hydro hóa của ngành công nghiệp hóa chất dầu khí (Ma và nnk., 2016; Li và nnk., 2012). Tài nguyên niken có nguồn gốc từ hai loại: quặng sunfua và quặng laterit, lần lượt chiếm khoảng 30\% và $70 \%$ trữ lượng niken thế giới. Tuy nhiên, hiện nay lượng niken sản xuất ra chiếm trên $60 \%$ sản lượng có nguồn gốc từ quặng niken sunfua (Bai và nnk., 2010) vì niken trong quặng sunfua dễ tuyển

*Tác giả liên hệ

E- mail: trantrungtoi@humg.edu.vn DOI: 10.46326/JMES.2021.62(3b).05 làm giàu bằng các phương pháp tuyển truyền thống: tuyển nổi (tương tự tuyển pyrit), hoặc tuyển từ kết hợp tuyển nổi. Do đó, quặng niken sunfua đang dần cạn kiệt, quặng niken laterit có hàm lượng niken thấp (Pournaderi và nnk., 2014) dần trở thành nguồn tài nguyên chính để sản xuất niken (Kim và nnk., 2010; Pickles, 2013; Pickles, 2014).

Trong quặng laterit, niken phân bố ở nhiều khoáng vật khác nhau, nhưng giàu nhất trong khoáng nontronit, serpentin và các khoáng sắt oxit (goethit/ limonit) (Al - Khirbash, 2015; Rice, 2016). Ngoài ra, quặng này có hàm lượng niken thấp $(0,5 \div 2,5 \% \mathrm{Ni})$, thường được chia thành ba lớp: limonit, saprolit và garnierit (Agacayak và nnk., 2016; Zevgolis và nnk., 2010). Vì vậy, các phương pháp tuyển truyền thống để nâng cao 
hàm lượng niken đều không hiệu quả (Quast và nnk., 2015).

Xét về lợi ích, quặng niken laterit có hai lợi thế phát triển đáng kể: thứ nhất, chúng có trữ lượng dồi dào; thứ hai, quặng này hình thành ngay trên mặt đất, dễ khai thác (Bunjaku và nnk., 2012; Pickles và Elliott, 2015). Đây là điều kiện thuận lợi để phát triển công nghệ chế biến sâu loại quặng này (Ma và nnk.,2016; Elliott và nnk., 2015). Để đáp ứng nhu cầu ngày càng tăng về niken và giúp khai thác loại quặng này hiệu quả hơn, thì điều quan trọng là phát triển quy trình xử lý quặng niken laterit hiệu quả. Bài báo này tổng hợp các nghiên cứu về việc phát triển các quy trình xử lý quặng niken laterit trong những năm gần đây, đồng thời đề xuất định hướng công nghệ xử lý quặng niken laterit ở Thanh Hóa, Việt Nam.

\section{Quy trình thủy luyện xử lý quặng niken laterit}

Các quy trình thủy luyện để xử lý quặng niken laterit bao gồm: thiêu hoàn nguyên - hòa tách amoniac; hòa tách axit áp suất cao; hòa tách axit môi trường khí quyển và hòa tách đống. Nguyên tắc cơ bản của các quy trình này là hòa tan niken và coban trong quặng niken laterit bằng dung môi amoniac $\left(\mathrm{NH}_{3}-\left(\mathrm{NH}_{4}\right)_{2} \mathrm{CO}_{3}\right)$ hoặc axit $\left(\mathrm{H}_{2} \mathrm{SO}_{4}, \mathrm{HCl}\right.$, $\mathrm{HNO}_{3}$ ), sau đó xử lý dung dịch để thu hồi Niken và coban.

\subsection{Thiêu hoàn nguyên - hòa tách amoniac}

Đây được gọi là quy trình Caron (Hình 1). Nó phù hợp cho việc xử lý quặng limonit ở nhiệt độ thiêu $700 \div 800^{\circ} \mathrm{C}$ với sự có mặt của chất khử; tiếp đó là quá trình hòa tách niken trong sản phẩm sau thiêu trong dung môi amoniac - amoni cacbonat. Kết quả có thể hòa tách $75 \div 80 \% \mathrm{Ni}$ và $40 \div 60 \%$ Co.

Chen và nnk. (2010) đã nghiên cứu xử lý quặng niken laterit (Ni 1,17\%, Fe 45,56\%) ở điều kiện thiêu tối ưu như sau: khối lượng chất khử $10 \%$, thời gian 120 phút, nhiệt độ $750 \div 800^{\circ} \mathrm{C}$. Còn trong quá trình hòa tách amoniac, tỷ lệ lỏng/rắn là $4 / 1(\mathrm{~mL} / \mathrm{g})$, nhiệt độ $40^{\circ} \mathrm{C}$, thời gian 120 phút và tỷ lệ nồng độ của $\mathrm{NH}_{3} / \mathrm{CO}_{2}$ là $90 / 60$ $(\mathrm{g} / \mathrm{L})$. Trong điều kiện này, hiệu suất tách niken và coban lần lượt là $86,25 \%$ và $60,84 \%$.

Valix và nnk. (2002) đã sử dụng nguyên tố $S$ trong nghiên cứu và cho thấy sự có mặt của $\mathrm{S}$ có thể làm giảm nhiệt độ thiêu hoàn nguyền xuống

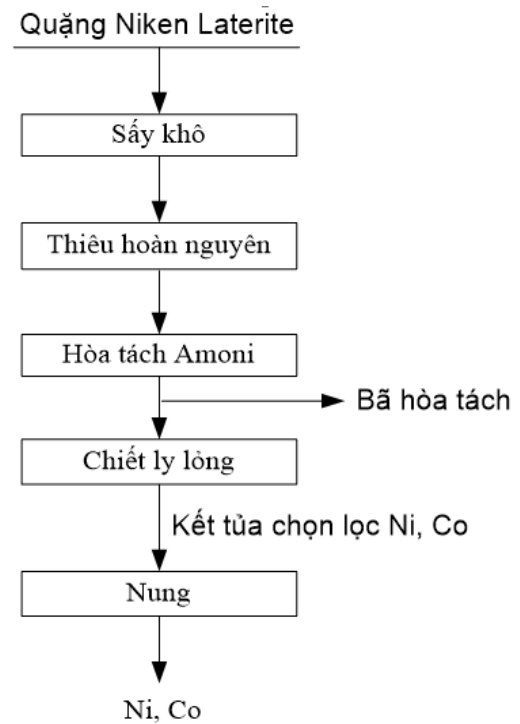

Hình 1. Quy trình công nghệ Caron.

$600^{\circ} \mathrm{C}$ mà vẫn cho khả năng thu hồi Ni cao hơn từ quặng limonite và saprolit. Việc bổ sung 5\% S trong quá trình thiêu đã đạt được mức thu hồi niken $98 \%$ từ quặng saprolit và $80,6 \%$ từ quặng limonit. Trong khi không bổ sung $\mathrm{S}$, độ thu hồi $\mathrm{Ni}$ lần lượt là $28 \%$ và $29 \%$ từ các quặng này.

Tóm lại, niken và coban có thể được tách đồng thời bằng quá trình thiêu hoàn nguyên - hòa tách amoniac. Tuy nhiên, tỷ lệ thu hồi niken và coban

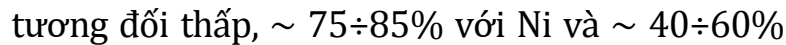
với Co. Ngoài ra, quá trình này không thể sử dụng để xử lý quặng niken laterit có hàm lượng đồng cao, bởi vì đồng có thể tạo phức với amoniac như niken và coban dẫn đến việc tách niken và coban khó khăn. Quy trình này cũng không phù hợp để xử lý quặng niken laterit có hàm lượng silic và magiê cao. So với các quy trình thủy luyện khác để xử lý loại quặng này, hạn chế của quá trình thiêu hoàn nguyên - hòa tách amoniac là yêu cầu tiêu thụ năng lượng cao hơn. Trong những năm gần đây, các nhà nghiên cứu đã cải tiến để công nghệ này cải thiện việc chiết tách Ni lên $80 \div 85 \%$. Vì vậy, quy trình thiêu hoàn nguyên - hòa tách amoniac trở thành một điểm nổi bật và đã được triển khai áp dụng vào thực tế.

\subsection{Hòa tách axit áp suất cao}

Hòa tách axit áp suất cao là quá trình hòa tách trực tiếp quặng trong thiết bị kín Autocla, bao gồm ba khâu: chuẩn bị, hòa tách và thu hồi niken, coban từ dung dịch (Hình 2). Quá trình thường tiến hành ở nhiệt độ $250 \div 270^{\circ} \mathrm{C}$, áp suất $4 \div 5 \mathrm{MPa}$ 


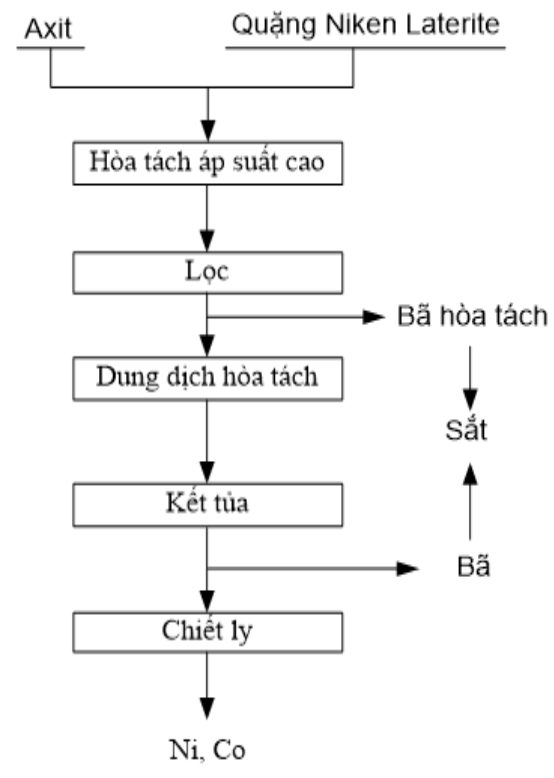

Hình 2. So đồ quy trình hòa tách axit áp suất cao.

và nồng độ axit cao (yêu cầu axit tự do $25 \div 100$ $\mathrm{g} / \mathrm{L}$ ) để hòa tan niken và coban lớn hơn $90 \%$. Do đó, quá trình này yêu cầu về chi phí bảo trì thiết bị cao, thường xuyên phải kiểm soát các điều kiện của quy trình. Ngoài ra, phương pháp này không thích hợp với quặng niken larterit chứa hàm lượng magiê cao, vì magiê sẽ làm tăng lượng axit tiêu thụ.

Axit được sử dụng nhiều nhất là axit sunfuric (Önal và nnk, 2014; Johnson và nnk., 2005; Rubisov và nnk., 2000). Nghiên cứu của Johnson và nnk. (2005) cho thấy, việc tách niken từ quặng laterit giàu nontronit được tăng cường bằng cách tăng axit hoặc bổ sung một lượng nhỏ natri vào dung dịch hòa tách. Với chi phí axit là $380 \mathrm{~kg} / \mathrm{tấ}$ quặng, niken và coban hòa tách lần lượt là 93,3\% và $91,6 \%$. Trong khi dung môi hòa tách cho thêm $5 \mathrm{~g} / \mathrm{L}$ ion natri thì chỉ cần chi phí axit $320 \mathrm{~kg} /$ tấn quặng, hiệu suất hòa tách niken và coban lần lượt là $93,6 \%$ và $95,2 \%$. Kết quả chỉ ra rằng có thể giảm được ít nhất $15 \%$ axit khi thêm $5 \mathrm{~g} / \mathrm{L}$ ion natri vào dung dịch hòa tách. Điều này có lợi trong việc giảm chi phí trung hòa axit vì nồng độ axit tự do cuối cùng thấp hơn trong quá trình xử lý thu hồi.

Các ứng dụng thực tế của phương pháp hòa tách axit sulfuric áp suất cao để xử lý quặng niken laterit của Moa ở Cuba và Ramu ở Papua New Guinea. Quặng niken laterit Ramu, thành phần chính 1,20\% Ni, 0,1\% Co, 45\% Fe; hòa tách ở điều kiện: mật độ bùn $32 \%$, kích thước hạt $4 \div 200 \mu \mathrm{m}$, nhiệt độ hòa tách $255^{\circ} \mathrm{C}$, áp suất trong Autocla 4,8
MPa. Với thời gian hòa tách $50 \div 60$ phút, hiệu suất hòa tan niken và coban cao, tương ứng 96\% và 94\% (Yang., 2013). Tuy nhiên, có một số vấn đề trong quá trình sản xuất: (1) Khó kiểm soát một số tạp chất trong quá trình hòa tách áp suất cao, điều này sẽ ảnh hưởng đến sản phẩm niken và coban cũng như dẫn đến tăng chi phí để loại bỏ tạp trong quá trình sản xuất. (2) Sản phẩm niken và coban có độ ẩm cao làm tăng chi phí vận chuyển lên $20 \%$, ảnh hưởng đến hiệu quả kinh tế của toàn bộ quá trình sản xuất.

Các nghiên cứu gần đây đang thay thế axit sunfuaric bằng axit nitric và axit clohydric trong quá trình hòa tách. Hòa tách áp suất cao sử dụng axit nitric (NAPL) đã được cấp bằng sáng chế vào năm 2008. Wang và nnk. (2008) đã chỉ ra rằng axit nitric có thể được sử dụng làm dung môi hòa tách quặng niken laterit có chứa magiê cao. Chi tiết quá trình này như sau: Thứ nhất, quặng niken laterit magiê cao được nghiền đến cỡ hạt $75 \mu \mathrm{m}(\geq 80 \%)$; thứ hai, hỗn hợp axit nitric và quặng niken laterit với một tỷ lệ axit/rắn nhất định được cho vào thiết bị hòa tách Autocla trong điều kiện nghiên cứu khảo sát (nồng độ axit nitric $500 \div 1500 \mathrm{~kg} /$ tấn quặng, tỷ lệ lỏng/rắn là $3 / 1 \div 10 / 1$, nhiệt độ $120 \div 200^{\circ} \mathrm{C}$, thời gian hòa tách $30 \div 120$ phút, tốc độ khuấy $300 \div 500$ vòng/phút); thứ ba, tinh chế và loại bỏ sắt bằng cách kết tủa thông qua việc thêm $\mathrm{MgCl}_{2}$ làm chất trung hòa và kiểm soát độ $\mathrm{pH}$ từ 2,5 $\div 3,5$ dẫn đến nồng độ sắt thấp hơn $0,07 \mathrm{~g} / \mathrm{L}$ trong dung dịch sau hòa tách; thứ tư, kết tủa thu hồi đồng thời niken và coban trong dung dịch khử sắt ở $\mathrm{pH}$ từ $6,5 \div 8,0$ và cũng sử dụng $\mathrm{MgCl}_{2}$ làm chất trung hòa. Kết quả hiệu suất thu hồi niken và coban đều đạt 95\%, còn magie đạt 98\%. Trong quá trình hòa tách, axit nitric vừa có tác dụng hòa tan niken và coban, lại vừa oxy hóa sắt (II) lên sắt (III), rất hữu ích cho việc hình thành hematit theo phản ứng từ (1) đến (4) và dẫn đến nồng độ sắt trong dung dịch hòa tách thấp. Bã hòa tách còn lại có hàm lượng sắt cao, không có lưu huỳnh, thích hợp làm nguyên liệu để luyện gang.

$$
\begin{gathered}
\mathrm{FeOOH}_{(\text {rắn) }}+3 \mathrm{H}^{+} \rightarrow \mathrm{Fe}^{3+}+2 \mathrm{H}_{2} \mathrm{O} \\
\mathrm{Fe}_{3} \mathrm{O}_{4(\text { rắn })}+8 \mathrm{H}^{+} \rightarrow 2 \mathrm{Fe}^{3+}+\mathrm{Fe}^{2+}+4 \mathrm{H}_{2} \mathrm{O} \\
3 \mathrm{Fe}^{2+}+\mathrm{NO}_{3}+4 \mathrm{H}^{+} \rightarrow 3 \mathrm{Fe}^{3+}+\mathrm{NO} \uparrow+2 \mathrm{H}_{2} \mathrm{O} \\
2 \mathrm{Fe}^{3+}+3 \mathrm{H}_{2} \mathrm{O} \rightarrow \mathrm{Fe}_{2} \mathrm{O}_{3(\text { rắn })}+6 \mathrm{H}^{+}
\end{gathered}
$$


Zhang và nnk. (2015) đã sử dụng quá trình hòa tách chọn lọc bằng axit clohydric có áp suất để tách niken và coban từ quặng laterit saprolitic chứa $1,37 \% \mathrm{Ni}$ và $18,8 \% \mathrm{Fe}$. Kết quả cho thấy hòa tách áp suất bằng axit clohydric là một phương pháp hiệu quả để hòa tan niken từ quặng laterit. Các điều kiện hòa tách tối ưu như sau: nồng độ $\mathrm{HCl}$ $350 \mathrm{~g} / \mathrm{L}$, tỷ lệ $\mathrm{L} / \mathrm{R}$ là 1,0 , nhiệt độ $150^{\circ} \mathrm{C}$ và thời gian là 90 phút. Trong điều kiện này, hiệu suất hòa tách niken và coban lần lượt là $89,4 \%$ và $97,3 \%$. Đối với giai đoạn đầu của quá trình hòa tách, sắt sẽ hòa tan và kết tủa theo phản ứng (5) *(7). Vì vậy, quá trình hòa tách áp suất bằng axit clohydric có tính chọn lọc tốt đối với niken.

$$
\begin{gathered}
\mathrm{FeOOH}_{(\text {răn })}+4 \mathrm{HCl} \rightarrow \mathrm{FeCl}_{4}^{-}+2 \mathrm{H}_{2} \mathrm{O}+\mathrm{H}^{+} \\
\mathrm{FeCl}_{4}^{-}+2 \mathrm{H}_{2} \mathrm{O} \rightarrow \mathrm{FeOOH}+3 \mathrm{H}^{+}+4 \mathrm{Cl}^{-} \\
2 \mathrm{FeOOH} \rightarrow \mathrm{Fe}_{2} \mathrm{O}_{3}+\mathrm{H}_{2} \mathrm{O}
\end{gathered}
$$

\subsection{Hòa tách axit áp suất thưò̀ng}

Cũng giống như quá trình hòa tách axit áp suất cao, axit được sử dụng nhiều nhất trong quá trình này là axit sunfuric (MacCarthy và nnk., 2014). Và cũng có nhiều nghiên cứu về việc sử dụng axit clohydric, axit nitric và axit hữu cơ làm dung môi hòa tan để tách niken từ quặng niken laterit (Guo, 2015; Astuti và nnk., 2015; Wang và nnk., 2012).

Kursunoglu và Kaya (2016) đã nghiên cứu hòa tách niken, coban và sắt từ quặng laterit của Caldag bằng axit sulfuric ở áp suất thường. Hiệu suất hòa tan niken, coban và sắt tương ứng là $91,9 \%, 93,5 \%$ và $80,5 \%$ ở điều kiện hòa tách: Kích thước hạt quặng $212 \mu \mathrm{m}$, nồng độ axit sulfuric 2 $\mathrm{mol} / \mathrm{L}$, thời gian hòa tách là 6 giờ, mật độ bùn $10 \%$, nhiệt độ hòa tách ở $90^{\circ} \mathrm{C}$ và tốc độ khuấy 500 vòng/phút.

Wang và nnk. (2012) đã nghiên cứu đặc điểm hòa tách axit clohydric ở áp suất thường đối với quặng laterit có hàm lượng niken thấp (Ni 0,82\%, Fe 49,92\%, Co 0,078\%). Các điều kiện hòa tách tối uu như sau: tỷ lệ axit/quặng là 1,25 , lỏng/rắn là 4, nhiệt độ hòa tách là $80^{\circ} \mathrm{C}$ và thời gian hòa tách là 2 giờ. Hiệu suất hòa tách $\mathrm{Ni}, \mathrm{Co}$ và $\mathrm{Fe}$ trong các điều kiện này lần lượt là 95,1\%; 99,0\% và 94,6\%. Mức độ hòa tan niken của các khoáng chất giảm dần theo thứ tự sau: siderite > chrysotile > magnetit $>$ maghemite $>$ goethite $>$ hematit $>$ chromite $\approx$ ringwoodite.

McCarthy và nnk. (2014) đã giới thiệu quy trình hòa tách niken trực tiếp bằng axit nitric ở áp suất thường và có thể sử dụng cho tất cả các loại quặng niken laterit. Quặng được khai thác và nghiền nhỏ dưới $2 \mathrm{~mm}$. Sau đó, quặng được trộn với axit nitric đạt hàm lượng rắn $20 \div 30 \%$ và được đưa đến các bể ủ nóng ở $110^{\circ} \mathrm{C}$. Thời gian hòa tách từ $2 \div 6$ giờ (thường là 4 giờ). Quá trình này chứng minh đơn giản và an toàn khi hoạt động liên tục (Hình 3).

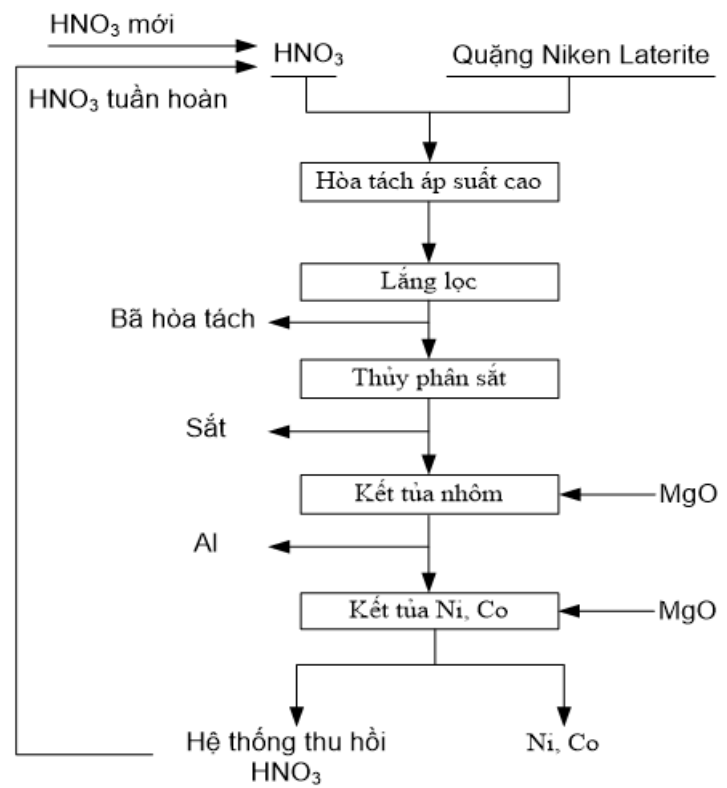

Hình 3. So đồ quy trình hòa tách niken trực tiếp bằng axit $\mathrm{HNO}_{3}$ (McCarthy và nnk., 2014).

Axit hữu cơ thân thiện môi trường hơn axit vô cơ nên cũng được sử dụng trong nghiên cứu gần đây (Astuti và nnk., 2015; 2016). Astuti và cộng sự đã sử dụng axit xitric làm chất hòa tách ở áp suất thường đối với quặng saprolitic ở Indonesia có hàm lượng niken $1,76 \%$. Hiệu suất hòa tách niken cao nhất đạt 95,6\% ở điều kiện hòa tách: Kích thước hạt quặng $1212 \div 355 \mu \mathrm{m}$, nồng độ axit tối ưu $1 \mathrm{~mol} / \mathrm{L}$, thời gian hòa tách 15 ngày, mật độ bùn $20 \%$, nhiệt độ hòa tách $40^{\circ} \mathrm{C}$ và tốc độ khuấy 200 vòng/phút.

Các kết quả nghiên cứu cho thấy, quá trình hòa tách axit áp suất cao đạt được một số kết quả như: tỷ lệ tách niken/ coban cao và hiệu suất cao; nhưng cũng có một số nhược điểm: do điều kiện áp suất, môi trường axit trong quá trình hòa tách và loại bỏ tạp chất phức tạp, nên quá trình hòa tách axit áp suất cao phải sử dụng nhiều thiết bị 
đặc chủng riêng, chi phí đầu tư cao. Ngoài ra, cần có lượng axit tự do dư thừa để hòa tách niken và coban càng nhiều càng tốt. Hơn nữa, quá trình hòa tách axit sunfuric áp suất cao chỉ thích hợp để xử lý quặng laterit limonit có hàm lượng nhôm thấp và magie thấp. Còn, quá trình hòa tách axit nitric có áp suất và quá trình hòa tách axit clohydric chọn lọc ở áp suất cao vẫn chưa hoàn thiện và cần được tối ưu hóa thêm.

Khi so sánh với quá trình hòa tách axit áp suất cao, quá trình hòa tách axit ở áp suất thường có chi phí thấp, thiết bị đơn giản, nhưng hiệu suất hòa tách niken tương đối thấp, nên có thể thích hợp để hòa tách quặng niken laterit có hàm lượng niken thấp (Kursunoglu và nnk., 2016). Quá trình hòa tách axit hữu cơ thân thiện với môi trường hơn quá trình hòa tách axit vô cơ, nhưng thời gian hòa tách axit hữu cơ rất lâu.

\section{Quy trình hỏa luyện xử lý quặng niken laterit}

Phương pháp hỏa luyện để xử lý quặng niken laterit bao gồm: sản xuất feronickel bằng quá trình nung khử trong lò quay - nấu chảy bằng lò điện và sản xuất sten niken trong quá trình luyện phản ứng (Warner., 2006). Các phương pháp hỏa luyện hầu hết thích hợp với quặng laterit saprolitic và granit có chứa niken cao (Zevgolis và nnk., 2010; Li và nnk., 2011). Niken và sắt trong quặng bị khử thành kim loại, sau đó sản phẩm feronickel được tách ra khỏi xỉ thông qua quá trình nấu chảy. Sản xuất sten niken trong quá trình nấu chảy khử là một kiểu tương tự như quá trình sản xuất feroniken, chỉ khác là có cho thêm lưu huỳnh vào nguyên liệu trong quá trình nấu.

Quá trình khử bằng lò quay - nấu chảy bằng lò điện được gọi là RKEF, với các bước chính là sấy, nung, hoàn nguyên và nấu chảy. Lưu trình RKEF được mô tả ở Hình 4 (Keskinkilic, 2012).

Keskinkilic và nnk. (2012) đã khảo sát đặc điểm nung của quặng niken laterit (Ni 1,26\%, Fe $32,6 \%)$. Quá trình gồm hai khía cạnh: loại bỏ nước liên kết hóa học trong quá trình chuyển hóa goethit - hematit; và loại bỏ các chất bay hơi trong khoảng $350 \div 700^{\circ} \mathrm{C}$. Do đó, nên chọn $700^{\circ} \mathrm{C}$ làm nhiệt độ nung để loại bỏ hiệu quả nước liên kết hóa học và tất cả các chất bay hơi. Zevgolis và nnk. (2010) đã nghiên cứu sự biến đổi pha của quặng laterit trong quá trình nung nóng sơ bộ và khử bằng cacbon monoxit. Sự biến đổi của goethit

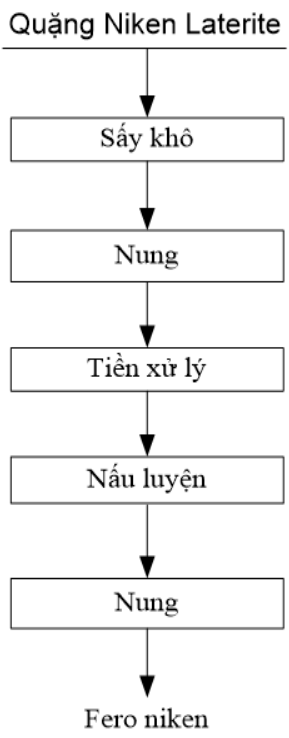

Hình 4. So đồ quy trình nấu feroniken tù quặng niken laterit.

thành hematit và sự phân hủy của clorit và serpentin, được xác định trong quá trình gia nhiệt sơ bộ. Quá trình hoàn nguyên để khử các hợp chất của sắt và niken sang dạng kim loại. Đối với sắt ở dạng goethit thì hiệu suất hoàn nguyên lên đến $95 \%$, trong khi quặng hematit hiệu suất hoàn nguyên chỉ đạt $50 \%$. Khả năng khử cao hơn có thể là do diện tích bề mặt riêng của loại quặng goethit cao hơn. Sau hoàn nguyên là quá trình nấu chảy sắt và niken vừa hoàn nguyên để tạo ra feroniken.

Điều quan trọng là kiểm soát quá trình nung hoàn nguyên trong lò quay, vì nó ảnh hưởng mạnh mẽ đến việc thu hồi niken và hàm lượng feroniken. Pournaderi và nnk. (2014) đã nghiên cứu ảnh hưởng của nhiệt độ, lượng than và thời gian hoàn nguyên đối với quặng laterit $(1,26 \% \mathrm{Ni}$, $32,6 \% \mathrm{Fe}$ ) sau nghiền sơ bộ. Các nghiên cứu chỉ ra rằng, mức độ hoàn nguyên ra sắt kim loại bị giới hạn ở nhiệt độ dưới $900{ }^{\circ} \mathrm{C}$ và tăng nhanh ở nhiệt độ cao hơn. Còn mức độ hoàn nguyên của Ni và Co tăng khi tăng nhiệt độ từ $700 \div 800^{\circ} \mathrm{C}$, rồi gần như chững lại ở nhiệt độ hoàn nguyên $900^{\circ} \mathrm{C}$ và sau đó lại tăng lên khi tăng nhiệt đến $1100{ }^{\circ} \mathrm{C}$. Sắt có một vai trò kép ở nhiệt độ hoàn nguyên cao: làm tăng tốc độ phản ứng Budoa và đồng thời có thể phản ứng với các oxit Ni và Co thông qua phản ứng (8) và (9) để tạo ra các nguyên tố kim loại. Việc tăng lượng than không ảnh hưởng đến mức độ hoàn nguyên sắt ở $900{ }^{\circ} \mathrm{C}$, nhưng có ảnh hường đến mức độ hoàn nguyên niken và không ảnh hưởng đến mức độ hoàn nguyên của Co ở tất cả các nhiệt 
độ. Ở 1100 C, các phản ứng khử diễn ra nhanh và gần như kết thúc trong vòng 2400 giây; nhưng phản ứng cần thời gian dài hơn để hoàn thành ở $1000^{\circ} \mathrm{C}$.

$$
\begin{aligned}
& \mathrm{NiO}+\mathrm{Fe}=\mathrm{Ni}+\mathrm{FeO} \\
& \mathrm{CoO}+\mathrm{Fe}=\mathrm{Co}+\mathrm{FeO}
\end{aligned}
$$

Quá trình nung hoàn nguyên trong lò quay nấu chảy bằng lò điện, cần phải thực hiện nấu chảy ở $^{1500 \div 1600}{ }^{\circ} \mathrm{C}$, dẫn đến tiêu thụ năng lượng cao. Do đó tính thương mại của phương pháp này bị hạn chế và chỉ phù hợp để xử lý quặng laterit chứa niken hàm lượng cao, nhằm đảm bảo hiệu quả kinh tế.

Các quy trình hỏa luyện có ưu điểm ngắn gọn, đơn giản và gần như đã hoàn thiện. Tuy nhiên có nhược điểm: khó tách riêng được coban ra khỏi sản phẩm; yêu cầu quặng laterit chứa hàm lượng niken cao và tiêu thụ năng lượng đáng kể. Vì vậy, để giảm tiêu thụ năng lượng của các quá trình hỏa luyện, đòi hỏi quặng cần phải được tuyển làm giàu trước.

\section{Quy trình nung hoàn nguyên - tuyển từ}

Các quy trình thủy luyện rất phức tạp và mất nhiều thời gian (Zheng và nnk., 2014), nhưng chi phí năng lượng thấp. Ngược lại, các quy trình hỏa luyện đơn giản nhưng tiêu tốn nhiều năng lượng và yêu cầu quặng laterit có hàm lượng niken cao (Ma và nnk., 2016). Để khắc phục hạn chế của quy trình hỏa luyện, trong những năm gần đây, các nhà khoa học đã đề xuất thay thế khâu nấu chảy bằng khâu tuyển từ để thu hồi các hạt feroniken sinh ra trong giai đoạn hoàn nguyên. Nung hoàn nguyên tuyển từ là một quy trình xử lý quặng niken laterit mới và đầy hứa hẹn, có tiềm năng ứng dụng cao vào thực tiễn, do quy trình này đơn giản và tiêu thụ ít năng lượng và có thể xử lý được các loại quặng niken laterit khác nhau (Jiang và nnk., 2013; Tang và nnk., 2014). Trong giai đoạn nung hoàn nguyên, niken và sắt được khử thành kim loại ở nhiệt độ $1000 \div 1200^{\circ}$ C. Quặng sau nung hoàn nguyên được nghiền mịn và đem tách các hạt feroniken bằng phương pháp tuyển từ ướt; tỷ lệ thu hồi niken đạt trên $90 \%$.

Quá trình tuyển từ mục đích làm tăng hàm lượng niken trong sản phẩm feroniken. Niken phải được khử thành kim loại càng nhiều càng tốt và sắt phải được khử hạn chế để đạt được hiệu quả khử niken có chọn lọc và thu được sản phẩm feroniken với hàm lượng niken cao. Tuy nhiên, để đạt được cả hai mục tiêu cùng một lúc là rất khó, vì sắt sẽ được khử thành kim loại ở một mức độ nào đó và có chức năng như một chất mang (Pickles và Elliott, 2015). Ngoài ra, điều quan trọng là phải tăng được kích thước hạt feroniken hình thành để hợp kim feroniken có thể được tách hiệu quả và chọn lọc bằng phương pháp tuyển từ. Zhu và nnk. (2012) đã nghiên cứu quá trình nung hoàn nguyên quặng laterit limontic và quặng laterit saprolitic. Kết quả cho thấy, khi trong nguyên liệu có một lượng khoáng silicat phù hợp, sẽ giúp ngăn cản sự hoàn nguyên sắt và tăng mức độ hoàn nguyên niken. Đồng thời, việc bổ sung canxi sulfat cũng thúc đẩy sự hình thành và phát triển kích thước hạt feroniken trong quá trình hoàn nguyên. Ngoài ra, hàm lượng và tỷ lệ thu hồi của niken rất nhạy cảm với chi phí chất khử. Jiang và nnk. (2013) đã sử dụng quy trình nung hoàn nguyên - tuyển từ để xử lý quặng laterit chứa nhiều sắt $(1,49 \% \mathrm{Ni}, 34,69 \% \mathrm{Fe})$, với việc bổ sung natri sunfat. Kết quả thu được sản phẩm feroniken có hàm lượng 9,87\% $\mathrm{Ni}$ và tỷ lệ thu hồi niken đạt 90,90\% ở nhiệt độ hoàn nguyên $1200^{\circ} \mathrm{C}$ trong 50 phút với việc bổ sung $10 \% \mathrm{Na}_{2} \mathrm{SO}_{4}$ và $2 \%$ than. Elliott và nnk. (2015) đã nghiên cứu quá trình hoàn nguyên chọn lọc quặng limonit có hàm lượng niken là $1,38 \%$ và hàm lượng sắt là $45,70 \%$. Họ sứ dụng quy trình tăng nhiệt và hoàn nguyên hai giai đoạn để tạo ra sản phẩm feroniken. Quy trình nung quặng ban đầu với $6 \%$ than và bổ sung $4 \%$ lưu huỳnh ở $600^{\circ} \mathrm{C}$ trong 1 giờ, tiếp theo là tăng trưởng kích thước hạt ferroniken trong 1 giờ ở $1000^{\circ} \mathrm{C}$. Sản phẩm feroniken thu được với hàm lượng niken là $4 \%$ và mức thu hồi niken đạt được là 93,2\%. Ngoài ra, kích thước hạt ferroniken tăng lên khi tăng nhiệt độ và thời gian. Năm 2015, Elliott cũng nghiên cứu ảnh hưởng của việc bổ sung than đá, lưu huỳnh, pyrit và natri sulphat và nhiệt độ hoàn nguyên trong khoảng $1000 \div 1200^{\circ}$ C đối với sự khử có chọn lọc của laterit limonite và saprolit. Kết quả cho thấy rằng việc bổ sung lưu huỳnh vào limonite phù hợp hơn với lưu huỳnh vào saprolit. Hơn nữa, việc bổ sung cacbon và lưu huỳnh tăng lên và nhiệt độ hoàn nguyên cao hơn sẽ dẫn đến sự hình thành các hợp kim feroniken chứa hàm lượng niken thấp. Đối với mẫu limonite có bổ sung lưu huỳnh 4\% ở nhiệt độ hoàn nguyên $1100^{\circ} \mathrm{C}$ trong 1 giờ, kích thước hạt feroniken 
trung bình là 1,59 $\mu \mathrm{m}$, so với 1,01 $\mu \mathrm{m}$ đối với cùng một loại quặng và điều kiện khử mà không bổ sung lưu huỳnh. Trong trường hợp của saprolit, việc bổ sung lưu huỳnh dường như làm giảm kích thước hạt feroniken trung bình. Do đó, các nghiên cứu này nhắc nhở rằng trong quá trình tối ưu hóa các điều kiện khử có chọn lọc, việc lựa chọn các chất phụ gia thích hợp cho các loại quặng niken laterit khác nhau là rất quan trọng. Zhou và nnk. (2015) tiến hành khử quặng laterit niken hàm lượng thấp với việc bổ sung $\mathrm{Na}_{2} \mathrm{~S}, \mathrm{Na}_{2} \mathrm{SO}_{4}$ và $\mathrm{CaSO}_{4}$. So sánh ba chất phụ gia, nhận thấy $\mathrm{Na}_{2} \mathrm{SO}_{4}$ thúc đẩy sự phát triển của feroniken và cải thiện hàm lượng của niken trong feroniken là tốt nhất.

Như vậy, quá trình hoàn nguyên chọn lọc phụ thuộc phần lớn vào hai nhóm yếu tố: kiểm soát môi trường hoàn nguyên (phụ thuộc vào loại chất khử, chi phí chất khử) và lựa chọn phụ gia thích hợp. Các chất phụ gia thích hợp có thể tăng cường quá trình nung khử chọn lọc, ức chế quá trình khử sắt, tăng kích thước của các hạt feroniken và cải thiện hiệu quả tuyển tách từ về sau. Sự hình thành và phát triển kích thước của các hạt feroniken trong giai đoạn nung hoàn nguyên là rất quan trọng, vì nó quyết định việc tách hiệu quả các hạt feroniken khỏi các tạp chất trong giai đoạn tuyển từ tiếp theo. Điều này bị chi phối bởi nhiệt độ hoàn nguyên, thời gian cũng như các chất phụ gia phù hợp. Quá trình hoàn nguyên niken có chọn lọc và sự phát triển kích thước hạt feroniken quyết định mức độ thu hồi niken cũng như chất lượng sản phẩm feroniken thu được.

\section{Quy trình xử lý quặng niken laterit trong nước}

Theo báo cáo "Tài nguyên Khoáng sản Việt Nam" (Trị và nnk., 2000), mỏ cromit Cổ Định là một trong các mỏ có tài nguyên trữ lượng Co và $\mathrm{Ni}$ lớn, có nguồn gốc từ vỏ phong hóa ở đỉnh và sườn dãy Núi Nưa. Quá trình địa chất trong kỷ Đệ tứ đã tạo nên các thân quặng cromit, thân quặng Co và Ni trong các trầm tích aluvi, proluvi nằm ở các thung lũng xung quanh dãy Núi Nưa. Trải qua 60 năm hoạt động khai thác cromit, tại khu vực Cổ Định, đã hình thành nên các bãi thải quy mô rất lớn (khoảng $17 \div 18$ triệu tấn). Các loại bùn thải được chôn lấp tại chỗ, hiện phân bố rộng khắp trong các khai trường ở các khu mỏ Mỹ Cái, Cổ Định và Mậu Lâm, Tĩnh Mễ. Trong quá trình khai thác và tuyển cromit, Co và Ni không được quan tâm. Với các dạng tồn tại như đã xác định, Co và Ni nằm nguyên trong các loại bùn thải, tạo nên các thân quặng, có nguồn gốc thứ sinh. Hàm lượng Ni trong bùn và trong quặng nguyên khai (lấy từ các mỏ và sản phẩm phong hóa) biến động trong phạm vi khá lớn từ $0,24 \div 0,95 \%$; hàm lượng Co biến động trong khoảng 0,014 $0,58 \%$. Ở khu Mậu Lâm giàu Co và Ni hơn khu Mỹ Cái. Trong đó cấp hạt thô +2 mm giàu Co và Ni hơn cấp hạt mịn. Đặc biệt, các kết vón laterit trong bùn thải rất giàu Co.

Cho đến hiện nay, bãi thải này vẫn chưa có định hướng công nghệ phù hợp để thu hồi các kim loại có giá trị, trong đó có Ni. Có một vài nghiên cứu mang tính chất trao đổi định hướng là chính. Cơ và nnk. (2007), đã nghiên cứu về đặc tính và dạng tồn tại của niken chứa trong mẫu quặng cromit, từ đó đã áp dụng nhiều phương pháp xử lý như: dùng phương pháp tuyển nổi; nung sulfua hoá quặng rồi đưa vào tuyển nổi; nung thiên tích sau đem tuyển nổi; nung từ hoá quặng sau đem tuyển từ và dùng phương pháp thuỷ luyện để chuyển niken vào dung dịch khi hoà tách. Trong số các phương pháp tuyển cơ giới đã nêu trên, chỉ nâng hàm lượng niken lên khoảng $1,17 \div 2,67 \% \mathrm{Ni}$, với mức thực thu nằm trong giới hạn $17,28 \div$ $35,31 \%$. Chỉ có phương pháp thuỷ luyện là có triển vọng, ở điều kiện thí nghiệm: mẫu nghiền đến $100 \%$ cấp $-0,074 \mathrm{~mm}$, dùng dung môi axit $\mathrm{H}_{2} \mathrm{SO}_{4} 2 \%$, $\left(\mathrm{NH}_{4}\right)_{2} \mathrm{SO}_{4} 15 \mathrm{~g} / \mathrm{L}$; nhiệt độ hoà tách $250^{\circ} \mathrm{C}$ và thời gian hoà tách 2 giờ, cho phép hòa tan được $90,5 \%$ Ni và $88,2 \%$ Co vào dung dịch. Đề tài mới chỉ dừng lại ở khâu hoà tách, chưa triển khai được khâu thu hồi Coban và Niken ra khỏi dung dịch. Khanh và nnk. (2010) trong quá trình nghiên cứu hoàn thiện công nghệ tuyển quặng cromit có nghiên cứu tuyển thu hồi niken từ đuôi thải. Theo báo cáo này, thành phần khoáng của đuôi thải chủ yếu là sét nontronit, antigorit (một biến thể của serpentin), thạch anh, geothit/ limonit. Thành phần cấp hạt của đuôi thải gồm: cấp $+2 \mathrm{~mm}$ chiếm $11 \div 16 \%$ và cấp $-0,053 \mathrm{~mm}$ chiếm $40 \div 45 \%$. Quá trình nghiên cứu theo hai hướng: Nung sulfua hóa - tuyển nổi; và thiêu hoàn nguyên - tuyển từ. Kết quả quy trình nung hoàn nguyên - tuyển từ là tốt nhất, thu được tinh quặng chứa hàm lượng $\mathrm{Ni}>1,2 \%$ với mức độ thu hồi đạt 65\%, thu hoạch 34\%. Tinh quặng thu được sau tuyển từ lại đem hòa tách trong dung dịch amoni rồi kết tủa tạo niken carbonat. Từ đó, tiếp tục hòa tách trong dung dịch axit sunfuric rồi kết tủa và 
làm sạch để thu được sản phẩm muối $\mathrm{NiSO}_{4} \cdot 6 \mathrm{H}_{2} \mathrm{O}$ đạt độ sạch 99\% với mức thực thu chung đạt $84 \div 85 \%$. Tuy nhiên, nghiên cứu này mới chỉ tập chung thu hồi niken, mà chưa tận thu tổng hợp cả sắt và coban.

Ngoài ra, còn có một vài đề tài nghiên cứu về đối tượng quặng này, tuy nhiên chủ yếu tập trung nghiên cứu tuyển cromit để nâng cao hiệu suất thu hồi và hàm lượng crom trong tinh quặng, còn đối với coban và niken nằm trong đuôi tuyển thì chỉ nêu định hướng thải riêng để thu hồi coban - niken sau này khi có công nghệ tuyển phù hợp.

Như vậy, nhận thấy rằng, niken tồn tại trong bùn thải của quá trình khai thác mỏ cromit như một dạng thân quặng thứ sinh, thuộc dạng quặng niken laterit. Loại quặng này chứa hàm lượng niken thấp $(0,24 \div 0,95 \% \mathrm{Ni})$ không phù hợp xử lý bằng phương pháp hỏa luyện hay thủy luyện. Các công trình nghiên cứu tuyển bằng phương pháp truyền thống cơ bản không đạt hiệu quả. Quy trình nung hoàn nguyên - tuyển từ bước đầu đã đạt được hiệu suất thu hồi niken $65 \%$. Đây là quy trình thân thiện môi trường, có chi phí thấp và phù hợp với xu hướng phát triển chung của các nước trên thế giới như Trung Quốc, Indonesia, ... Tuy nhiên, khi áp dụng quy trình này, cần lưu ý tận thu đồng thời cả các kim loại $\mathrm{Fe}$ - $\mathrm{Ni}$ - Co để làm nguyên liệu cho sản xuất hợp kim niken - coban hoặc hợp kim feroniken hàm lượng thấp, như vậy mới tăng được hiệu quả kinh tế và tăng tính khả thi khi triển khai vào thực tiễn.

\section{Kết luận}

Các quy trình luyện kim thủy luyện có ưu điểm là thu hồi tối đa các kim loại niken, coban, sắt và magiê từ quặng laterit; nhưng nhược điểm là dài và phức tạp. Sự phát triển của quy trình hòa tách axit $\mathrm{HNO}_{3}$; axit $\mathrm{HCl}$ trong những năm gần đây, đặc biệt là việc hòa tách axit nitric là khá hiệu quả để xử lý quặng niken laterit. Tuy nhiên, quá trình này mới nghiên cứu ở quy mô phòng thí nghiệm, việc ứng dụng vào thực tế cần xem xét kỹ hơn. Hỏa luyện là quy trình đơn giản, tuy nhiên lại tiêu thụ nhiều năng lượng. Vì vậy các quy trình hỏa luyện khó ứng dụng vào thực tế. Nung hoàn nguyên tuyển từ là quy trình mới phát triển, có thể xử lý được các loại quặng niken laterit khác nhau; không yêu cầu nhiệt độ cao để nấu chảy và quy trình đơn giản. Vì vậy, quy trình này có thể sẽ được ứng dụng rộng rãi để xử lý quặng niken laterit trong tương lai.

\section{Những đóng góp của tác giả}

Tác giả Trần Trung Tới hình thành ý tưởng, cấu trúc bài báo và hoàn thiện bản thảo cuối cùng; tác giả Phùng Tiến Thuật hoàn thiện nội dung 2, nội dung 3; tác giả Nguyễn Tuấn Khanh hoàn thiện nội dung 4, nội dung 5.

\section{Tài liệu tham khảo}

Al - Khirbash S., (2015). Ore Geol. Rev. 65, 199 212.

Agacayak T., Zedef V. and Aras A., (2016). J. Cent. South Univ. 23, 39 - 43.

Astuti W., Hirajima T., Sasaki K. and Okibe N., (2015). Miner. Metall. Proc. 32, 176 - 185.

Astuti W., Hirajima T., Sasaki K. and Okibe N., (2016). Hydrometallurgy 161, 138 - 151.

Bunjaku A., Kekkonen M., Pietilä K. and Taskinen P., (2012). Trans. Inst. Min. Metall. C. 156 - 165.

Cơ V. T, Hoàn C. V; Hà V. V; Linh N. B (2007). Nghiên cứu khả năng thu hồi Niken trong quặng mỏ crômit Cổ Định, Thanh Hoá, đề tài Bộ, Bộ Công Thương.

Chen S., Guo X., Shi W. and Li D., (2010). Journal of Central South University of Technology 17.

Elliott R., (2015a). A Study on the Role of Sulphur in the Thermal Upgrading of Nickeliferous Laterite Ores, Ph.D. thesis, Queen's University.

Elliott R., Rodrigues F., Pickles C. A. and Peacey J., (2015b). Can. Metall. Quart. 54, 395 - 405.

Guo Q., Qu J., Han B., Zhang P., Song Y. and Qi T., (2015). Miner. Eng. 71, 1 - 6.

Johnson J. A., McDonald R. G., Muir D. M. and Tranne J., (2005). Hydrometallurgy 78, 264 270.

Jiang M., Sun T., Liu Z., Kou J., Liu N. and Zhang S., (2013). Int. J. Miner, 2013. Process. 123, 32 - 38.

Khanh $\mathrm{H}$. V và nnk. (2010). Kết quả nghiên cứu hoàn thiện quy trình công nghệ tuyển và sử dụng hợp lý quặng cromit Cổ Định, Thanh Hóa. Viện khoa học và Công nghệ Mỏ - Luyện kim, Mã số 2038 
Kursunoglu S. and Kaya M., (2016). Int. J. Miner. Process. 150, 1 - 8.

Kim J., Dodbiba G., Tanno H., Okaya K., Matsuo S. and Fujita T., (2010). Miner. Eng. 23, 282 - 288.

Keskinkilic E., Pournaderi S., Geveci A. and Topkaya Y. A., (2012). J. S. Afr. I. Min. Metall. $112,877,882$.

Li G., Shi T., Rao M., Jiang T. and Zhang Y., (2012). Miner. Eng. 32, 19 - 26.

Li B., Wang H. and Wei Y., (2011). Miner. Eng. 24, $1556-1562$.

Li J., Xiong D., Chen H., Wang R. and Liang Y., (2012). Hydrometallurgy 129, 14 - 18.

Lu J., Liu S., Shangguan J., Du W., Pan F. and Yang S., (2013). Miner. Eng. 49, 154 - 164.

Liu P., Li B., Cheung S. C. and Wu W., (2016). Appl. Therm. Eng. 109, 542 - 559.

Lv X., Bai C., He S. and Huang Q., (2010). ISIJ Int. 50, 380 - 385.

Liu Z., Sun T., Wang X. and Gao E., (2015). Int. J. Miner. Metall. Mater. 22, 901 - 906.

Ma X., Cui Z. and Zhao B., (2016). JOM 68, 3006 3014

Ma B., Wang C., Yang W., Yang B. and Zhang Y., (2013). Miner. Eng. 45, 151 - 158.

MacCarthy J., Addai - Mensah J. and Nosrati A., (2014). Miner. Eng. 69, 154 - 164.

Önal M. A. R. and Topkaya Y. A., (2014). Hydrometallurgy 142, 98 - 10.Pickles C. A., Harris C.T., Peacey J. and Forster J., (2013). Miner. Eng. 54, 52 - 62.

Pickles C. A., Forster J., and Elliott R., (2014). Miner. Eng. 65, 33 - 40.

Pickles C. A., Forster J., and Elliott R., (2014). Miner. Eng. 65, 33 - 40.

Pickles C. A. and Elliott R., (2015). Trans. Inst. Min. Metall. C. 124, 208 - 216.

Pournaderi S., Keskinkılıç E., Geveci A. and Topkaya Y. A., (2014). Can. Metall.Quart. 53, 26 - 37.
Quast K., Connor J. N., Skinner W., Robinson D. J. and Addai - Mensah J., (2015). Miner. Eng.

Rao M., Li G., Zhang X., Luo J., Peng Z. and Jiang T., (2016). Sep. Sci. Technol. 51, 1408 - 1420.

Rao M., Li G., Zhang X., Luo J., Peng Z. and Jiang T., (2016). Sep. Sci. Technol. 51, 1727 - 1735.

Rubisov D. H., Krowinkel J. M. and Papangelakis V. G., (2000). Hydrometallurgy 58, 1 - 11.

Rice N. M., (2016). Miner. Eng. 88, 28 - 52.Tang X., Liu R., Yao L., Ji Z., Zhang Y. and Li S., (2014). International Journal of Minerals, Metallurgy, and Materials 21, 955 - 961.

Trị T. V, Tiến Ph. C, \& Lâm T. Q., (2000). Tài nguyên Khoáng sản Việt Nam. Cục Địa chất và Khoáng sản Việt Nam - Viện nghiên cứu Địa chất và Khoáng sản.

Valix M. and Cheung W. H., (2002). Miner. Eng. 15, $523-530$.

Wang B., Guo Q., Wei G., Zhang P., Qu J. and Qi T., (2012). Hydrometallurgy 129, 7 - 13.

Wang C., Yin F., Chen Y., Yang Y., Yang B., Gao W., Wang Z., Yuan S., Wang J., Wang N., Li D. and Jie X., (2008). CN Patent No. 101,289,704.

Warner A. E., Diaz C. M., Dalvi A. D., Mackey P. J. and Tarasov A. V., (2006). JOM 58, 11 - 20.

Yang. S., (2013). Nonferrous Met. (Extr. Metall). 1, 003

Zhang P., Guo Q., Wei G., Meng L., Han L., Qu J. and Qi T (2015). Hydrometallurgy 157, 149-158

Zevgolis E. N., Zografidis C., Perraki T. and Devlin E., Therm J., (2010). Anal. Calorim. 100, 133 139.

Zhou S., Li B., Wei Y., Wang H., Wang C. and Ma B (2015). "Effect of Additives on Phase Transformation of Nickel Laterite Ore During Low- Temperature Reduction Roasting Process Using Carbon Monoxide," in Drying, Roasting, and Calcining of Minerals, edited by P. B. Thomas et al. (TMS, 2015), pp. 177-184

Zhu D. Q., Cui Y., Vining K., Hapugoda S., Douglas J., Pan J. and Zheng G. L., (2012). Int. J. Miner. Process. 106, 41 - 50. 